\title{
Outcomes of anterior levator resection in congenital blepharoptosis
}

\begin{abstract}
Purpose To identify any consistent factors which may predict over- or undercorrection of congenital blepharoptosis treated by anterior levator resection.

Methods A retrospective case note review of 100 consecutive patients undergoing anterior levator resection for congenital blepharoptosis was performed to identify: (1) the amount of ptosis and degree of levator function present pre-operatively and (2) the surgical outcome. For unilateral ptosis, a successful result was defined as a lid level within $1 \mathrm{~mm}$ of the fellow lid following a single operation. Results Seventy-six per cent of all unilateral cases had a successful outcome at 6 weeks following surgery, falling slightly to $74 \%$ by 6 months. The most common complications at 6 months were undercorrection $(19 \%)$ and overcorrection (7\%). All patients undercorrected at 6 months had a preoperative levator function in the range of 4-10 mm with a mean of $6.4 \mathrm{~mm}$, whereas all those overcorrected at 6 months had a levator function in the range $\mathbf{9 - 1 5} \mathbf{~ m m}$ with a mean of $12.2 \mathrm{~mm}$. In the whole population, the degree of levator function was a predictor of increased risk of overcorrection, with a trend towards predicting undercorrection as well. Conclusions In this series of patients, preoperative levator function was found to be the most significant predictor of surgical outcome for anterior levator resection.
\end{abstract}

Key words Congenital blepharoptosis, Levator function, Levator resection

Surgery for blepharoptosis in congenital levator dystrophy is acknowledged to have an unpredictable outcome. ${ }^{1-3}$ Anterior levator resection is the standard approach used to correct ptosis in those patients with at least $4 \mathrm{~mm}$ of levator palpebrae superior muscle function. ${ }^{4}$ The amount of levator resected at surgery is determined by a number of factors assessed pre-operatively including levator function, degree of ptosis and the presence of normal eye movements. Attempts have been made to relate accurately the amount of surgery required for a successful surgical outcome to these factors, most notably by Beard and Berke. $^{5,6}$

Berke suggested a method using the preoperative levator function to determine the level at which the lid is set during surgery, disregarding the exact amount of levator resected. ${ }^{6}$ An analysis of large numbers of surgical results was used to determine the recommended placement of the lid margin. If the levator function equals $8 \mathrm{~mm}$, then the lid is set at the desired height. With a levator function of less than $8 \mathrm{~mm}$ the lid is set higher than the required height, anticipating a fall in the lid position post-operatively. With a levator function of more than $8 \mathrm{~mm}$, the lid is set lower because the lid position will rise subsequently if levator function is good. The method, whilst undoubtedly useful, relies upon a consistent effect of anaesthesia on muscle tone and consequent lid position per-operatively, and on having the operated eye reliably placed in the primary position during surgery. Both of these may be difficult variables to control.

Beard, by contrast, devised a formula which bases the amount of levator resection on both the levator function and the degree of ptosis. ${ }^{5}$ Each is graded into three categories with levator function either good, fair or poor and ptosis either mild, moderate or severe. In cases with either poor levator function and severe ptosis, or good levator function and mild ptosis, alternative surgical techniques to levator resection are recommended. This approach necessitates delaying surgery until accurate preoperative measurements can be made.

Both Beard's and Berke's recommendations have proved useful guidelines for surgery. However, it is well recognised that accurate prediction of surgical outcome remains difficult. Berlin, in a series of 58 congenital ptosis cases, using advancement of the levator aponeurosis alone without Muller's muscle, found that $69 \%$ had an acceptable result (defined as a lid level within $1 \mathrm{~mm}$ of the desired level) at 6 weeks. ${ }^{3}$ This dropped to $52 \%$ with an average follow-up of 4.6 months. Similarly in another large series of 228 patients with congenital ptosis only $43 \%$ had lid levels within $1 \mathrm{~mm}$ of each other at a year or more following one surgical procedure, with an additional $28 \%$ having lid levels within 
1-2 mm of each other. ${ }^{1}$ With this degree of unpredictability, any factors which could be identified to predispose to imperfect surgical results would be helpful in assessing and treating individual patients. In Berlin's series the difference in outcome between smaller and larger amounts of ptosis was not statistically significant, although there was a trend toward better outcome for smaller degrees of ptosis. ${ }^{3}$ The amount of levator function was not found to affect results.

Undercorrections outnumbered undercorrections by 10:1 and a pre-operative drop in lid height between 2 and 4 months following surgery was identified as a major cause. This the authors attributed to the use of absorbable sutures. In the second series of patients overcorrection was also found to be the major post-operative problem, ascribed to poorer pre-operative levator function and a greater degree of ptosis in this group. ${ }^{1}$

We reviewed the surgical results of 100 consecutive cases of congenital levator dystrophy and attempted to identify consistent factors predicting poor surgical outcome.

\section{Methods}

\section{Patients}

One hundred consecutive cases (108 lids) of anterior levator resection undertaken for congenital levator dystrophy were reviewed retrospectively. All procedures were carried out either by or under direct supervision of one of us (A.G.T.). Patients were included in the study if they met both the following criteria: (1) congenital ptosis without associated ocular motility disturbance,

blepharophimosis or Marcus Gunn phenomenon and (2) levator function greater or equal to $4 \mathrm{~mm}$. Patients who had undergone previous levator surgery elsewhere were included, as were those with bilateral ptosis.

Pre-operative data collected included age and sex of the patient. Measurements of the margin reflex distance were recorded to assess pre- and post-operative lid levels. ${ }^{7}$ The amount of ptosis was recorded as the difference in millimetres between the upper margin reflex distances between the two eyes. In patients with bilateral ptosis, the amount of ptosis was recorded as the difference between the normal palpebral fissure width $(10 \mathrm{~mm})$ and the measured width. In none of these patients was the lower eyelid considered to be at an abnormal level. A record was made of the level of the skin crease and presence of a Bell's phenomenon where possible. A successful surgical result was defined as the lid levels within $1 \mathrm{~mm}$ of each other post-operatively.
During the study period, 100 patients (57 male, 43 female) underwent surgery. The age range at surgery was 3 to 60 years with a median of 7 years. Eight patients underwent bilateral anterior levator resections, whilst 92 underwent unilateral surgery. Sixteen of the latter group had undergone prior levator surgery elsewhere. Ninetysix patients had 6 weeks of follow-up and 75 of these were re-examined at 6 months post-operatively. Of the remaining 4 patients, 3 were seen 2 weeks post-operatively and subsequently lost to follow-up. One patient defaulted after the first post-operative visit at 2 days.

\section{Surgical technique}

The majority of the surgery was carried out under general anaesthesia owing to the predominance of children in the study. The intended skin crease was marked prior to local infiltration of 1-2 $\mathrm{ml}$ of $2 \%$ lignocaine with 1:200 000 adrenaline. A skin incision was made, deepened to the tarsal plate with scissors through the orbicularis muscle, and extended medially and laterally. The anterior tarsal surface was cleaned before dissection of the preseptal orbicularis muscle from the lower orbital septum. The orbital septum was opened to allow prolapse of the preaponeurotic fat pad and confirmation of the surgical planes. The levator aponeurosis and Muller's muscle were then detached from the upper border of the tarsal plate and separated from the conjunctiva until the anterior limit of the common sheath could be identified at the level of the superior conjunctival fornix. At this stage, the horns of the levator complex were cut if a levator resection of more than $13 \mathrm{~mm}$ was necessary to achieve a satisfactory lid height. Three long-acting absorbable sutures secured the levator muscle to the anterior tarsal surface, the lid height and curve being checked after placement of each. The sutures were adjusted if the lid height was felt to be unsatisfactory. Having secured the muscle, skin sutures were placed with deep bites taken through aponeurosis. A lower lid traction (Frost) suture was used with topical antibiotic ointment to protect the cornea before the eye was padded. ${ }^{8}$ The dressing and traction suture were removed either at 24 or $48 \mathrm{~h}$ postoperatively. The only variation from the above was in one patient with levator function greater than $13 \mathrm{~mm}$ in whom Muller's muscle was separated from levator and not advanced with the aponeurosis. Absolute adherence to the recommendations of Beard and Berke was not observed, although the amount of levator resection was guided by the degree of pre-operative ptosis and the peroperative lid level was set with regard to the documented levator function.

Table 1. Unilateral ptosis: post-operative results

\begin{tabular}{lccccc}
\hline & \multicolumn{2}{c}{ Results at 6 weeks } & & \multicolumn{2}{c}{ Results at 6 months } \\
\cline { 2 - 3 } \cline { 5 - 6 } & No. of lids & Success $(\%)$ & No. of lids & Success (\%) \\
\hline Primary surgery & 72 & $59(82)$ & 56 & 12 & $42(75)$ \\
Re-operations & 16 & $8(50)$ & $67(76)$ & 68 & $8(67)$ \\
Total & 88 & & $50(74)$ & 50 \\
\hline
\end{tabular}




\begin{tabular}{lccc}
\hline & No. of lids & Ptosis $(\mathrm{mm})$ & Levator function $(\mathrm{mm})$ \\
\hline Unilateral ptosis & 68 & $2.5 \pm 0.8$ & $8.4 \pm 2.9$ \\
Success & 50 & $2.5 \pm 0.7$ & $8.6 \pm 2.7$ \\
Undercorrection & 13 & $2.6 \pm 1.0$ & $6.4 \pm 1.8$ \\
Overcorrection & 5 & $2.2 \pm 0.6$ & $12.2 \pm 2.8$ \\
Primary surgery & 56 & $2.4 \pm 0.7$ & $8.4 \pm 2.7$ \\
Success & 42 & $2.4 \pm 0.6$ & $8.4 \pm 2.7$ \\
Undercorrection & 10 & $2.6 \pm 1.1$ & $7.0 \pm 1.6$ \\
Overcorrection & 4 & $2.3 \pm 0.7$ & $11.5 \pm 2.7$ \\
Re-operations & 12 & $2.7 \pm 1.0$ & $8.6 \pm 3.7$ \\
Success & 9 & $2.8 \pm 1.0$ & $9.4 \pm 2.7$ \\
Undercorrection & 3 & $2.7 \pm 1.2$ & $4.3 \pm 0.6$ \\
Overcorrection & 1 & 2.0 & 15.0 \\
\hline
\end{tabular}

Values are mean $\pm \mathrm{SD}$.

\section{Results}

One hundred and eight lids of 100 patients were treated with anterior levator resection. Ninety-two patients underwent unilateral surgery with an overall success rate of $76 \%$ of 88 patients seen at 6 weeks and $74 \%$ of 68 patients reviewed at 6 months (Table 1). Failure was most commonly attributable to undercorrection, with a rate of $16 \%$ (14 patients) at 6 weeks and $19 \%$ (13 patients) at 6 months. Overcorrections were much less common, occurring in $8 \%$ (7 patients) at 6 weeks and $7 \%$ (5 patients) at 6 months. In these patients without successful outcomes, the degree of pre-operative ptosis was not dissimilar from the successful group (Table 2). However, the levator function was found to be higher in the overcorrected group and lower, although less obviously, in the undercorrected group. The spread of pre-operative levator function versus outcome is depicted in Fig. 1. All patients undercorrected at 6 months had a pre-operative levator function in the range of $4-10 \mathrm{~mm}$ with a mean of $6.4 \mathrm{~mm}$, whereas all those overcorrected at 6 months had a levator function in the range of 9-15 mm with a mean of $12.2 \mathrm{~mm}$.

Other complications of unilateral ptosis surgery included corneal exposure in 3 patients, all of whom had a successful result of surgery. In 2 of these patients, topical lubrication was sufficient to control the symptoms and no patient required medication for longer than a year post-operatively. The remaining patient required two subsequent levator recession procedures but still achieved a successful cosmetic result following this and did not require lubrication. Conjunctival prolapse required excision in 1 patient and the lid curve was refashioned in 1 patient, both following successful levator surgery. Three patients underwent further

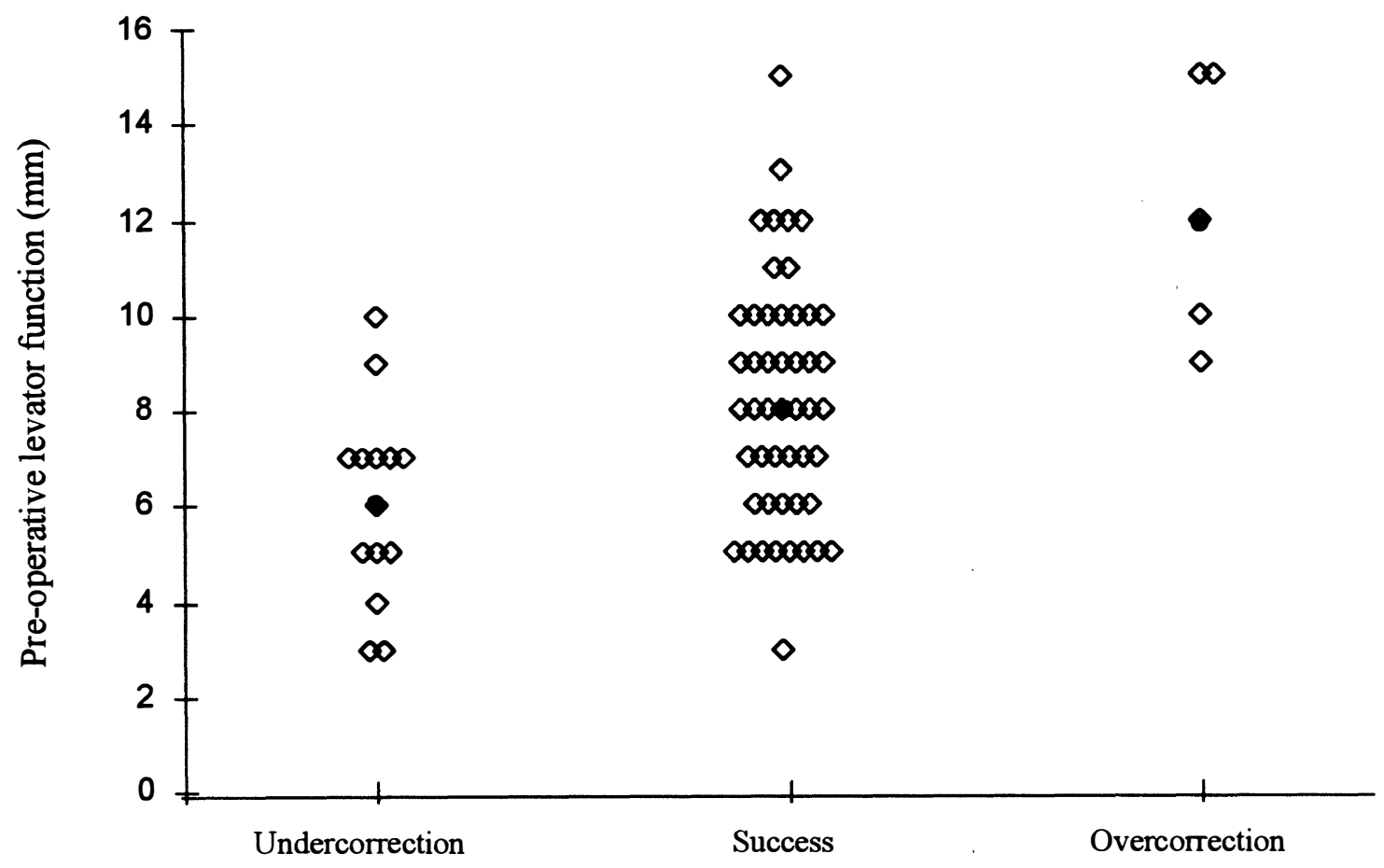

\section{Surgical Outcome}

Fig. 1. Scattergram showing pre-operative levator function against surgical outcome at 6 months. Filled circles indicate the mean. 


\begin{tabular}{lccccc}
\hline & \multicolumn{2}{c}{ Results at 6 weeks } & & \multicolumn{2}{c}{ Results at 6 months } \\
\cline { 2 - 3 } \cline { 5 - 6 } & No. of lids & Success $(\%)$ & & No. of lids & Success (\%) \\
\hline Primary surgery & 16 & $14(88)$ & & 14 & $9(64)$ \\
\hline
\end{tabular}

surgery to raise the skin crease, 2 following a successful result and 1 combined with surgery for an undercorrection. In total, 24 out of $92(26 \%)$ patients undergoing unilateral ptosis surgery required a further operation for the reasons detailed above.

After separating the 76 patients undergoing primary surgery for unilateral ptosis from those having redo procedures (16), the success rate improved to $82 \%$ at 6 weeks (of 72 patients reviewed) compared with $50 \%$ (of 16 patients reviewed) in the redo group. In the primary surgery group, the undercorrection rate was $14 \%$ (10 patients) at 6 weeks and $18 \%$ (10 patients) at 6 months. Corresponding overcorrection rates were $4 \%$ (3 patients) and $7 \%$ ( 4 patients). In the redo group both rates were higher, with $25 \%$ undercorrections at both 6 weeks ( 4 patients) and 6 months ( 3 patients), and $25 \%$ overcorrections (4 patients) at 6 weeks with a fall to $8 \%$ (1 patient) at 6 months. The levator function remained the most identifiable predictive factor for surgical failure in both subgroups (Table 2).

Eight patients had bilateral levator surgery. The outcome at 6 weeks showed $88 \%$ (14 lids) with a successful outcome with only 1 under- and 1 overcorrection. Follow-up was available at 6 months for 14 lids of which only $64 \%$ (9 lids) had a successful result, as there were 3 more undercorrections by this time (29\%) (Table 3). In this small group all 4 undercorrected lids had pre-operative levator functions of $\leqslant 7 \mathrm{~mm}$ and the 1 overcorrection was seen in a lid with levator function of $10 \mathrm{~mm}$. Five of the original 16 lids required further surgery. There were no other complications in this group.

\section{Discussion}

In this series of patients with congenital ptosis, the preoperative levator function was found to be the best predictor of surgical outcome. The amount of levator function was markedly higher in the overcorrected group. This attains statistical significance, but as this was a retrospective study it could not strictly be used to confirm any hypothesis, and so formal statistical analysis is not included. Similarly, patients with poorer levator function showed a tendency to be undercorrected. These trends are best appreciated in Fig. 1. By contrast, the influence of the degree of ptosis does not appear to be significant.
The importance of the levator function in determining the success rate agrees with the findings of Jordan and Anderson, ${ }^{1}$ who demonstrated more late undercorrections in patients with lesser degrees of levator function. However, this conclusion is not universal. Blomgren, using levator resection and Berlin, using aponeurosis advancement, found that the degree of pre-operative ptosis was more relevant to the outcome than levator function. ${ }^{3,9}$

The most common cause of failure was undercorrection (19\% at 6 months) rather than overcorrection ( $7 \%$ at 6 months), indicating that the amount of levator resection erred on the side of caution. Whilst overcorrection carries a higher risk of complications such as corneal exposure, it may be rectified using either massage, traction or further surgery, and should not deter the surgeon from aiming to correct the total degree of ptosis. These results support the widely held view that large resections of the upper lid retractors (levator aponeurosis with or without Muller's muscle) are required in patients with worse levator function. In these patients there is a tendency to undercorrect. Patients with good levator function require smaller resections to reduce the rate of overcorrection in this group.

\section{References}

1. Jordan DR, Anderson RL. The aponeurotic approach to congenital ptosis. Ophthalmic Surg 1990;21:237-44.

2. Hylkema HA, Koornneef L. Treatment of ptosis by levator resection with adjustable sutures via the anterior approach. $\mathrm{Br}$ J Ophthalmol 1989;73:416-8.

3. Berlin AJ, Vestal KP. Levator aponeurosis surgery: a retrospective review. Ophthalmology 1989;96:1033-7.

4. Beard C. The surgical treatment of blepharoptosis: a quantitative approach. Trans Am Ophthalmol Soc 1966;64:401-87.

5. Beard C. Ptosis. 3rd ed. St Louis: CV Mosby, 1981:84-183.

6. Berke RN. Types of operations indicated for congenital and acquired ptosis. In: Troutman R, Converse J, Smith B, editors. Plastic and reconstructive surgery of the eye and adnexa. Washington: Butterworth, 1962.

7. Sarver BL, Putterman AM. Marginal limbal distance to determine amount of levator resection. Arch Ophthalmol 1985;103:354-6.

8. Frost AD. Supporting suture in ptosis operations. Am J Ophthalmol 1934;17:633.

9. Blomgren I, Holmstrom H. Anterior levator resection in congenital genuine blepharoptosis. Scand J Plast Reconstr Surg 1986;20:189-95. 